\title{
The pentose phosphate pathway regulates chronic neuroinflammation and dopaminergic neurodegeneration
}

\author{
Dezhen Tu' ${ }^{1,2}$, Yun Gao ${ }^{1,2}$, Ru Yang ${ }^{1}$, Tian Guan', Jau-Shyong Hong ${ }^{2}$ and Hui-Ming Gao ${ }^{1,2^{*}}$ (D)
}

\begin{abstract}
Background: Metabolic dysfunction and neuroinflammation are increasingly implicated in Parkinson's disease (PD). The pentose phosphate pathway (PPP, a metabolic pathway parallel to glycolysis) converts glucose-6-phosphate into pentoses and generates ribose-5-phosphate and NADPH thereby governing anabolic biosynthesis and redox homeostasis. Brains and immune cells display high activity of glucose-6-phosphate dehydrogenase (G6PD), the ratelimiting enzyme of the PPP. A postmortem study reveals dysregulation of G6PD enzyme in brains of PD patients. However, spatial and temporal changes in activity/expression of G6PD in PD remain undetermined. More importantly, it is unclear how dysfunction of G6PD and the PPP affects neuroinflammation and neurodegeneration in PD.
\end{abstract}

Methods: We examined expression/activity of G6PD and its association with microglial activation and dopaminergic neurodegeneration in multiple chronic PD models generated by an intranigral/intraperitoneal injection of LPS, daily subcutaneous injection of 1-methyl-4-phenyl-1,2,3,6-tetrahydropyridine (MPTP) for 6 days, or transgenic expression of A53T a-synuclein. Primary microglia were transfected with G6PD siRNAs and treated with lipopolysaccharide (LPS) to examine effects of G6PD knockdown on microglial activation and death of co-cultured neurons. LPS alone or with G6PD inhibitor(s) was administrated to mouse substantia nigra or midbrain neuron-glia cultures. While histological and biochemical analyses were conducted to examine microglial activation and dopaminergic neurodegeneration in vitro and in vivo, rotarod behavior test was performed to evaluate locomotor impairment in mice.

Results: Expression and activity of G6PD were elevated in LPS-treated midbrain neuron-glia cultures (an in vitro PD model) and the substantia nigra of four in vivo PD models. Such elevation was positively associated with microglial activation and dopaminergic neurodegeneration. Furthermore, inhibition of G6PD by 6-aminonicotinamide and dehydroepiandrosterone and knockdown of microglial G6PD attenuated LPS-elicited chronic dopaminergic neurodegeneration. Mechanistically, microglia with elevated G6PD activity/expression produced excessive NADPH and provided abundant substrate to over-activated NADPH oxidase (NOX2) leading to production of excessive reactive oxygen species (ROS). Knockdown and inhibition of G6PD ameliorated LPS-triggered production of ROS and activation of NF-kB thereby dampening microglial activation.

Conclusions: Our findings indicated that G6PD-mediated PPP dysfunction and neuroinflammation exacerbated each other mediating chronic dopaminergic neurodegeneration and locomotor impairment. Insight into metabolicinflammatory interface suggests that G6PD and NOX2 are potential therapeutic targets for PD.

Keywords: Microglia, Neurodegeneration, Neuroinflammation, Pentose phosphate pathway, Glucose-6-phosphate dehydrogenase, NADPH oxidase, Oxidative stress, Metabolic disruption

\footnotetext{
* Correspondence: gaohm@nju.edu.cn

${ }^{1}$ MOE Key Laboratory of Model Animal for Disease Study, Model Animal Research Center, Institute for Brain Sciences, Nanjing University, 12 Xuefu Road, Nanjing 210061, Jiangsu Province, China

${ }^{2}$ Neurobiology Laboratory, National Institute of Environmental Health

Sciences/National Institutes of Health, Research Triangle Park, Durham, NC 27709, USA
}

(c) The Author(s). 2019 Open Access This article is distributed under the terms of the Creative Commons Attribution 4.0 International License (http://creativecommons.org/licenses/by/4.0/), which permits unrestricted use, distribution, and reproduction in any medium, provided you give appropriate credit to the original author(s) and the source, provide a link to the Creative Commons license, and indicate if changes were made. The Creative Commons Public Domain Dedication waiver (http://creativecommons.org/publicdomain/zero/1.0/) applies to the data made available in this article, unless otherwise stated. 


\section{Background}

Parkinson's disease (PD), the most common age-related neurodegenerative movement disorder, is characterized by progressive loss of dopamine (DA) neurons in the substantia nigra (SN) [1]. Unclear etiology, delayed diagnosis, no effective treatment, and clinical trial failures reinforce the need to identify new targets for drug discovery for PD treatment. Recently, emerging evidence has uncovered important roles of metabolic and inflammatory dysregulation in PD pathogenesis [2-5]. Neuroinflammation is a complex cascade of self-defensive response to injurious stimuli in the central nervous system (CNS). However, substantial evidence has demonstrated important contribution of neuroinflammation to PD pathogenesis. For instance, DNA polymorphisms or variations in multiple inflammatory cytokines and in the HLA (human leukocyte antigen) region that contains many immune-related genes are associated with increased risk for PD [6, 7]. The SN of PD patients and animal models showed microglial activation and accumulation of inflammatory mediators [5, 8, 9]. Moreover, inhibition of neuroinflammation correlates with less neuronal impairment in various PD models [10-12]. Therefore, to discern mechanism(s) flipping neuroinflammation from a beneficial physiological response to a chronic neurodegenerative one is urgent and of paramount importance.

Recently, increasing preclinical and clinical evidence has revealed glucose metabolism disturbance in PD [2, 1315]. Interestingly, several recent studies have shown dysregulation of the pentose phosphate pathway (PPP, a metabolic pathway parallel to glycolysis) in PD [2, 13]. The PPP mainly coordinates anabolic biosynthesis and redox homeostasis by controlling its intracellular products ribose-5-phosphate (the biosynthetic precursor of nucleotides) and NADPH rather than provides energy supply [16]. Glucose-6-phosphate dehydrogenase (G6PD), the rate-limiting enzyme of the PPP, is a ubiquitous enzyme, but its expression and activity vary over a 10-fold range, being lowest in the skeletal muscle and highest in the brain $[17,18]$. It has been reported that neurons preferentially metabolize glucose via the PPP [19]. Earlier measurement of G6PD in red blood cells in PD patients has yielded conflicting results claiming reduced or unaltered activity [20, 21]. A postmortem study of PD brains has detected an increase in NADPH production in the putamen (a brain region affected in PD) of late-stage cases; unexpectedly, the putamen of early-stage PD and the cerebellum of early- and late-stage PD display a reduction in G6PD [13]. Up to date, neither NADPH nor G6PD in the $\mathrm{SN}$ (the major disease region in PD) has been examined in patients or animal models of PD. Nevertheless, available evidence reveals dysregulation of the G6PD and the PPP in PD. Their possible pathogenic roles in PD warrant further investigation.
Oxidative stress is a major component and connector of metabolic disruption and neuroinflammation and serves as a key pathologic factor in neurodegenerative diseases including PD [2, 3, 10, 22]. NADPH generated in the PPP is a cofactor and a reducing agent used in anabolic reactions (e.g. synthesis of glutathione, lipid, and nucleic acid). On the other hand, under catalysis by activated NADPH oxidase (NOX2), NADPH provides electron to molecular oxygen to generate the freeradical superoxide. Over-activated NOX2 is a major source of oxidative stress under inflammatory condition and has been implicated as a novel therapeutic target for neurodegenerative diseases [10]. High energy requirement, high glucose consumption, adequate production of reactive oxygen species (ROS), low antioxidant defense, abundant oxidation-sensitive lipids, and a restricted renewal and regenerative capacity of neurons render the brain extremely susceptible to glucose metabolism disruption, oxidative insults, and inflammatory destruction. Therefore, it is important to investigate possible causal roles of PPP-mediated metabolism disturbance and neuroinflammation in PD. In the present study, we provided the first evidence that G6PDmediated metabolic dysfunction in the PPP in brain microglia and neuroinflammation exacerbated each other inducing chronic neurodegeneration and locomotor impairment, thus identifying a mechanistic basis for chronic PD progression.

\section{Materials and methods}

\section{Animals}

All animals were treated in strict accordance with the National Institutes of Health (Bethesda, MD, USA) Guide for Humane Care and Use of Laboratory Animal. All efforts were made to minimize the number of animals and their suffering. Both timed-pregnant Fisher 344 rats and $\mathrm{C} 57 \mathrm{BL} / 6 \mathrm{~J}$ mice were obtained from the Jackson Laboratory (Bar Harbor, ME). The intranigral and intraperitoneal injection of lipopolysaccharide (LPS, 0111:B4) and daily subcutaneous injection of 1-methyl4-phenyl-1,2,3,6-tetrahydropyridine (MPTP, $15 \mathrm{mg} / \mathrm{kg}$ ) for 6 consecutive days were performed as previously described [23, 24]. At desired time points after the injection, mice were anesthetized with fatal plus and then transcardially perfused with ice-cold PBS, followed by rapid freezing in liquid nitrogen or perfusion with $4 \%$ PBS-buffered paraformaldehyde. Fixed and frozen brains were used for immunohistochemistry and western blotting assay respectively.

\section{Cell cultures}

Primary neuronal and glial cultures

Mesencephalic neuron-glia cultures were prepared from the ventral mesencephalon of embryonic day 14 
\pm 0.5 Fischer 334 rats. Cultures were maintained in minimum essential medium (MEM) supplemented with $10 \%$ heat-inactivated fetal bovine serum (FBS) and $10 \%$ heat-inactivated horse serum (HS), $1 \mathrm{~g} / \mathrm{L}$-glucose, $2 \mathrm{mM}$ L-glutamine, $1 \mathrm{mM}$ sodium pyruvate, and $0.1 \mathrm{mM}$ nonessential amino acids. Seven days after seeding, the cultures were either treated with vehicle or $10 \mathrm{ng} / \mathrm{mL}$ LPS with or without pretreatment with various reagents as specified in the figure legend. This concentration of LPS was used in all in vitro studies. At the time of treatment, the neuron-glia cultures were made up of $\sim 12 \%$ microglia, $48 \%$ astrocytes, and $40 \%$ neurons of which $2.8-3.8 \%$ were tyrosine hydroxylase (TH)-immunoreactive (IR) neurons [25]. Rat neuron-glia cultures were used to examine effects of pharmacological inhibition of G6PD.

\section{Mixed-glia cultures and microglia-enriched cultures}

Mixed-glia cultures were prepared from whole brains of postnatal day 1 mice. Disassociated brain cells were seeded into 96-well plates $\left(10^{5}\right.$ cells/well $)$ or $175 \mathrm{~cm}^{2}$ flasks (3 brains/flask) and maintained in DMEM/F-12 supplemented with $10 \%$ FBS, $2 \mathrm{mM}$ L-glutamine, $1 \mathrm{mM}$ sodium pyruvate, and $0.1 \mathrm{mM}$ nonessential amino acids. The medium was changed every 3 days. When reaching confluence at 11-12 days after seeding, the cultures contained $\sim 80 \%$ glial fibrillary acidic protein (GFAP)-IR astrocytes and $\sim 20 \%$ ionized calcium-binding adapter molecule 1 (Iba1)-IR microglia and were used for treatment or preparation of microglia-enriched cultures. Microglia were separated from astrocytes by shaking the flasks containing confluent mixed-glia cultures for $1 \mathrm{~h}$ at $150 \mathrm{rpm}$. The purity of microglia-enriched cultures was greater than $98 \%$, as determined by immunostaining for Iba1 and GFAP [25].

\section{Reconstituted cell cultures}

Mouse microglia were directly plated on top of the existing mouse neuron-astrocyte cultures [25]. Briefly, leucineleucine methyl ester (LME; $1.5 \mathrm{mM}$; Sigma-Aldrich) was added to mesencephalic neuron-glia cultures 2 days after cell seeding to deplete microglia. Three days later, the cultures were changed back to fresh medium without LME. Six days after the initial seeding, the neuronastrocyte cultures contained about 54\% astrocytes, $45 \%$ neurons, and less than $1 \%$ microglia as determined by immunostaining for GFAP, neuron-specific nuclear protein (Neu-N; 1:4000; Chemicon, Temecula, CA), and Iba1. Highly enriched microglia that were transfected with G6PD siRNAs or scramble siRNA for $30 \mathrm{~h}$ were added to the existing neuron-astrocyte cultures. The reconstituted cultures were treated with LPS or vehicle $12 \mathrm{~h}$ later. Five days after LPS/vehicle treatment, degeneration of DA neurons was assessed.

\section{Immunohistochemistry and immunocytochemistry}

Immunohistochemistry and immunocytochemistry were performed as described previously [25] with antibodies specific for G6PD (1:2500; Abcam), TH (1:5000; Millipore), or Iba1 (1:5000; Wako Pure Chemicals). Briefly, paraformaldehyde-fixed floating brain sections or cell cultures were blocked with appropriate normal serum followed by incubation overnight at $4{ }^{\circ} \mathrm{C}$ with primary antibodies. After incubation with an appropriate biotinylated secondary antibody and then the Vectastain ABC reagents (Vector Laboratory, Burlingame, CA, USA), the bound complex was visualized by color development with 3,3'-diaminobenzidine. Images were recorded with a CCD camera and the ZEN 2.3 lite software (Molecular Devices). The number of TH-IR neurons in neuron-glia cultures and the $\mathrm{SN}$ of fourteen evenly spaced brain sections from a series of 48 sections that covered the entire SN was counted by two individuals blind to the treatment.

\section{Immunofluorescence}

Mouse brain sections and cell cultures grown in glass chambers were used for double-labeled immunofluorescence. G6PD antibody (1:2500; Abcam), in combination with an antibody specific for CD11b (1:500; Bio-Rad Laboratories), TH (1:2000; Millipore), Neu-N (1:4000; Chemicon, Temecula, CA), or GFAP (1:1000; LifeSpan BioScience), was used as indicated in the figure legend. Phosphorylated p65 antibody (1:2000; Cell signaling) was used to detect NF- $\mathrm{B}$ activation in microglia-enriched cultures. Brain sections or cell cultures were then incubated with Alexa-488 (green) and/or Alexa-594 (red) conjugated secondary antibodies (1:1000; Invitrogen). All fluorescent images were obtained with a Zeiss LSM 780 or 880 NLO laser scanning confocal microscope.

\section{Western blotting assay}

Whole cell proteins were extracted from cultured cells by using radioimmunoprecipitation assay (RIPA) lysis buffer ( $50 \mathrm{mM}$ Tris- $\mathrm{HCl}, \mathrm{pH} 8.0,150 \mathrm{mM} \mathrm{NaCl}, 5 \mathrm{mM}$ EDTA, 1\% NP-40, 0.5\% sodium deoxycholate, 0.1\% SDS, and protease inhibitor cocktail). Protein concentrations were determined by using the biocinchoninic acid assay (BCA, ThermoFisher). Protein samples were resolved on NuPAGE 4-12\% Bis-Tris gels (Life technologies), and immunoblot analyses were performed using antibodies against G6PD (1:5000; Abcam), TH (1:5000; Millipore), or Iba-1 (1:2500; Wako Pure Chemicals). An antibody against $\beta$-actin or GAPDH (1:5000; Cell Signaling Technology) was included to monitor loading errors.

\section{Nuclear and cytosolic fractionation}

Nuclear and cytosolic proteins were extracted at $4{ }^{\circ} \mathrm{C}$ from primary microglia using the NE-PER ${ }^{\mathrm{TM}}$ Nuclear and 
Cytoplasmic Extraction Reagents kit (ThermoFisher) following the manufacturer's instructions. The volume ratio of CER I, CER II, and NER was 200:11:100. Microglia pellets were fully suspended in CER I with vigorous vortex at the highest speed and incubated on ice for $10 \mathrm{~min}$. Ice-cold CER II was added into the mixture, followed by vigorous vortex for $5 \mathrm{~s}$ and incubation on ice for $1 \mathrm{~min}$. The cell lysis was centrifuged for $5 \mathrm{~min}$ at maximum speed in a microcentrifuge $(\sim 13,000 \times g)$. The supernatant (cytoplasmic extract) was immediately transferred to a prechilled tube and placed on ice before use or storage. After rinsed twice with CER I to avoid cytosolic protein contamination, nuclei were suspended in icecold NER with vigorous vortex for $15 \mathrm{~s}$ and incubated on ice for $40 \mathrm{~min}$ with continue vortex for $15 \mathrm{~s}$ every 10 min. Nuclear extracts, collected by centrifuge at maximum speed $(\sim 13,000 \times g)$ in a microcentrifuge for 10 min, were immediately transferred to a prechilled tube and placed on ice before use or storage. Nuclear and cytosolic extracts were used to examine NF- $\mathrm{B}$ activation by Western blotting assay using antibodies against phosphorylated or total NF- $\mathrm{kB}$ subunit p65 (1:2500, cell signaling). While anti-histone H3 antibody (1:2500, Abcam) was used to monitor loading errors of nuclear proteins, anti-GAPDH (1:5000, cell signaling) or antitubulin antibody (1:5000, ThermoFisher) was used to monitor loading errors of cytosolic proteins.

\section{High-affinity $\left[{ }^{3} \mathrm{H}\right] \mathrm{DA}$ uptake assay}

The uptake assay was performed as described [25]. Briefly, after being rinsed twice with warm Krebs-Ringer buffer (KRB; $16 \mathrm{mM}$ sodium phosphate, $119 \mathrm{mM} \mathrm{NaCl}$, $4.7 \mathrm{mM} \mathrm{KCl}, 1.8 \mathrm{mM} \mathrm{CaCl} 2,1.2 \mathrm{mM} \mathrm{MgSO}, 1.3 \mathrm{mM}$ EDTA, and $5.6 \mathrm{mM}$ glucose; $\mathrm{pH} 7.4$ ), neuron-glia cultures were incubated for $15 \mathrm{~min}$ at $37^{\circ} \mathrm{C}$ with $1 \mu \mathrm{M}$ $\left[{ }^{3} \mathrm{H}\right] \mathrm{DA}$ (30 Ci/mmol, NEN, Boston, MA, USA) in KRB. After being washed three times with ice-cold KRB, cells were solubilized in $1 \mathrm{M} \mathrm{NaOH}$, and radioactivity was counted with a liquid scintillation counter. Nonspecific uptake was determined in the additional presence of 20 HM GBR 12935 dihydrochloride (a DA transporter inhibitor; S9659, Sigma-Aldrich).

\section{Superoxide assay}

The production of superoxide was determined by measuring the superoxide dismutase (SOD)-inhibitable reduction of tetrazolium salt WST-1, as described previously with modifications [26]. Primary microglia $\left(1 \times 10^{5}\right.$ cells/well), grown overnight in clear 96-well plates, were pretreated with vehicle or G6PD inhibitors for $30 \mathrm{~min}$ or transfected with G6PD siRNAs or scramble siRNA for $30 \mathrm{~h}$ followed by treatment with LPS in phenol red-free medium. The cultures were incubated with WST-1 (1 $\mathrm{mM}$ ) with or without $800 \mathrm{U} / \mathrm{mL}$ SOD for $30 \mathrm{~min}$ at $37^{\circ} \mathrm{C}$, and the absorbance at $450 \mathrm{~nm}$ was read with a SpectraMax Plus microplate spectrophotometer (Molecular Devices, Sunnyvale, CA).

\section{Detection of intracellular ROS}

The production of intracellular ROS (iROS) was measured by the cell-permeable fluorescence probe chloromethyl-29, 79-dichlorodihydrofluorescein diacetate (CM-H ${ }_{2}$ DCFDA; Cat\# C6827; Invitrogen) or $2^{\prime}, 7^{\prime}$ Dichlorofluorescin Diacetate $\left(\mathrm{H}_{2}\right.$ DCFDA; Cat\# 287810; Calbiochem) as described [27]. Briefly, mixed-glia cultures in black 96-well plates were pretreated with vehicle or G6PD inhibitors for $30 \mathrm{~min}$ followed by treatment with LPS for $6 \mathrm{~h}$ in phenol red-free medium. After incubated with $10 \mu \mathrm{M} \mathrm{H} \mathrm{H}_{2}$ DCFDA for $1 \mathrm{~h}$ at $37^{\circ} \mathrm{C}$, the fluorescence density was read at $488 \mathrm{~nm}$ for excitation and $525 \mathrm{~nm}$ for emission using a SpectraMax Gemini XS fluorescence microplate reader (Molecular Devices). Alternatively, microglia-enriched cultures grown in glass chambers were transfected with G6PD siRNAs or scramble siRNA for $30 \mathrm{~h}$ or pretreated with vehicle/G6PD inhibitors for $30 \mathrm{~min}$ followed by treatment for 18 or 24 $h$ with LPS as described in the figure legend. The cultures were stained with $10 \mu \mathrm{M} C M-\mathrm{H}_{2}$ DCFDA or antibody against 4-hydroxynonenal (4-HNE; 1:500; Abcam). Brain sections were immune-stained for 3nitrotyrosine (3-NT; 1:500; Abcam). Fluorescent images were collected using Zeiss LSM 780 or 880 confocal microscope and analyzed by using ImageJ software.

\section{Measurement of tumor necrosis factor- $a$}

The level of tumor necrosis factor- $\alpha$ (TNF $\alpha)$ at $6 \mathrm{~h}$ after LPS treatment in the culture medium was measured with commercial ELISA kits (R\&D Systems) following the manufacturer's instructions.

\section{G6PD enzyme activity assay and NADPH measurement} Mouse mesencephalic neuron-glia cultures were treated with either vehicle or LPS. The activity of G6PD and the level of NADPH in cell lysis from the cultures were measured following the manufacturer's instructions using Glucose 6 Phosphate Dehydrogenase Assay Kit (Colorimetric; ab102529; Abcam) and NADP/NADPH Assay Kit (ab65349; Abcam), respectively.

\section{Transfection with siRNA}

Mouse microglia grown in 24-well/6-well plates were transfected with G6PD siRNAs (ThermoFisher, 4390843 and 4390771) or scramble siRNA for $6 \mathrm{~h}$ using Lipofectamine $^{\mathrm{TM}}$ RNAiMAX transfection reagent (ThermoFisher, 13778) and antibiotic-free Opti-MEM ${ }^{\circledR}$ I reduced serum medium following the manufacturer's instructions and previous publication [28]. The final concentration of siRNAs is $10 \mathrm{nM}$. The cultures were then changed to 
normal growth medium, and $24 \mathrm{~h}$ later, the knockdown efficiency was assessed by western blotting assay.

\section{Lactate dehydrogenase cytotoxicity assay}

Measurement of extracellular lactate dehydrogenase (LDH) was performed using LDH cytotoxicity detection kit (Colorimetric, Takara) following the manufacturer's instructions. Briefly, the reconstituted $2 \mathrm{X} \mathrm{LDH}$ assay buffer $(50 \mu \mathrm{L})$ and the supernatant $(50 \mu \mathrm{L})$ were mixed by gentle shaking for $30 \mathrm{~s}$ and incubated at room temperature for $30 \mathrm{~min}$. The reaction was stopped by adding $50 \mu \mathrm{L}$ of $2 \mathrm{~N} \mathrm{HCl}$, and the absorbance value at $490 \mathrm{~nm}$ was read.

\section{MTT assay for cell viability}

Thiazolyl blue tetrazolium bromide (MTT, $0.45 \mathrm{mg} / \mathrm{mL}$, Sigma-Aldrich) was added to cultures treated with 6aminonicotinamide (6-AN, Sigma-Aldrich) and dehydroepiandrosterone (DHEA, Sigma-Aldrich) for a desired period of time. After incubation for $2 \mathrm{~h}$ at $37^{\circ} \mathrm{C}$, cell medium was removed from the cultures. The precipitated formazan, a product of MTT by the action of mitochondrial dehydrogenases, was solubilized with dimethyl sulfoxide and quantified spectrophotometrically at $570 \mathrm{~nm}$.

\section{Rotarod behavior test}

The rotarod behavior test was performed on a Rotamex device (Columbus Instruments, Columbus, OH, USA). The start speed was $0.5 \mathrm{rpm}$, the acceleration rate was set to $0.5 \mathrm{rpm} / 10 \mathrm{~s}$, and the maximum speed was $50 \mathrm{rpm}$. The mice underwent three consecutive trials with 30 min interval. The latency time to fall was recorded and analyzed to evaluate the motor coordination of mice.

\section{Statistical analysis}

All values are expressed as the mean \pm SEM. Differences among means were analyzed by using one- or two-way ANOVA with treatment as the independent factors. When ANOVA showed significant differences, multiple comparisons between means were tested by Dunnett's, Turkey, or Sidak's multiple post hoc testing. In all analyses, the null hypothesis was rejected at the 0.05 level.

\section{Results}

\section{Sustained high activity of the PPP in PD models}

To investigate the role of the PPP in PD, we first detected expression and activity of G6PD, the ratelimiting enzyme of the PPP, in four in vivo mouse PD models generated by an intranigral or intraperitoneal injection of LPS, daily subcutaneous injection of MPTP for 6 days, or transgenic overexpression of A53T mutant $\alpha$-synuclein. Firstly, 1 year after an intraperitoneal LPS injection, mouse midbrains showed increased levels of G6PD, gp91 $1^{\text {phox }}$ (the catalytic subunit of NOX2), and Iba1 and a decreased level of $\mathrm{TH}$, indicating sustained upregulation of G6PD, chronic neuroinflammation, and dopaminergic neurodegeneration (Fig. 1a, b). Moreover, the mRNA level of G6PD was significantly increased at 2 weeks or 9 months after LPS injection as compared with age-matched saline-injected controls (Fig. 1c). Secondly, at 2 weeks after an intranigral injection of LPS, we detected dramatic G6PD upregulation in the $\mathrm{SN}$, and upregulated G6PD was mainly located in microglia, but not in astroglia or neurons (Fig. 1d). The LPS-injected SN also showed profound activation of microglia and astroglia as well as damages and loss of DA neurons compared with vehicle-injected SN (Fig. 1d). Thirdly, in a sub-acute MPTP model of PD with daily subcutaneous injection of MPTP for 6 days, mouse midbrains displayed sustained upregulation of G6PD, gp91 ${ }^{\text {phox }}$, and Iba1 as well as reduction in $\mathrm{TH}$ level at 3 and 7 months after the last MPTP injection (Fig. 1e, f). Fourthly, midbrains of 1 -year-old A53T mutant $\alpha$-synuclein transgenic mice showed increased levels of G6PD and gp $91^{\text {phox }}$ compared with age-matched wild-type mice (Fig. 1g).

Double-labeled immunofluorescence in neuron-glia cultures treated with LPS for $48 \mathrm{~h}$ showed dramatic G6PD upregulation in activated microglia but not neurons or astroglia. Double-stained images of G6PD and $\mathrm{CD} 11 \mathrm{~b}$ in vehicle-treated control cultures, CD11b ${ }^{-} /$ $\mathrm{DAPI}^{+}$neurons and astroglia, and $\mathrm{CD} 11 \mathrm{~b}^{+} / \mathrm{DAPI}^{+}$ microglia all showed weak G6PD staining (Fig. 1h). Western blotting assay and immunostaining detected a significant increase in the level of G6PD protein in microglia-enriched cultures treated with LPS for $24 \mathrm{~h}$ (Fig. 1i and Additional file 1: Figure S1) and mesencephalic neuron-glia cultures treated with LPS for 7 days (Additional file 1: Figure S1). We next measured the enzyme activity of G6PD and the level of NADPH (a major product of the PPP and a substrate of NOX2) in mesencephalic neuron-glia cultures treated with vehicle or LPS. LPS-treated cultures exhibited longlasting increases in G6PD activity and NADPH level compared with vehicle-treated cultures (Fig. 1 j, k). Collectively, in multiple PD models, both microglial activation and loss of nigral DA neurons had positive correlation with increases in the expression/activity of G6PD and the production of NADPH (Fig. 1a, b, d-h). The concurrent upregulation of G6PD and NOX2 suggested that microglia with elevated G6PD activity generated excessive NADPH and provided abundant substrate to over-activated NOX2 thereby inducing oxidative stress, neuroinflammation, and neurodegeneration. Thus, metabolic disturbance in the PPP might be involved in chronic neuroinflammation and PD neurodegeneration. 


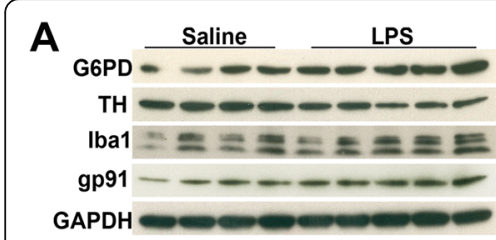

\section{D}

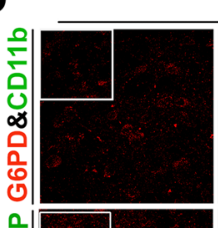

Control

H
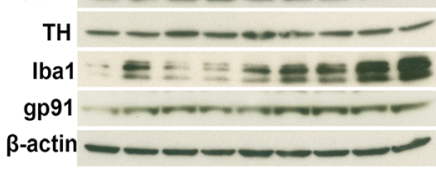

$\mathbf{F}$

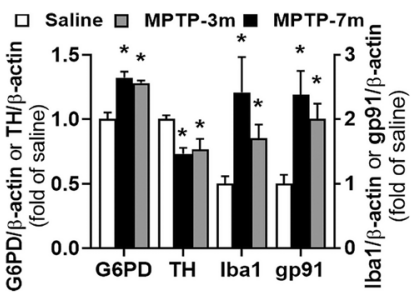

G

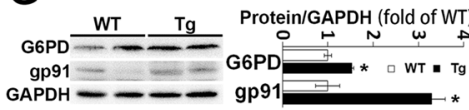

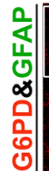

崩
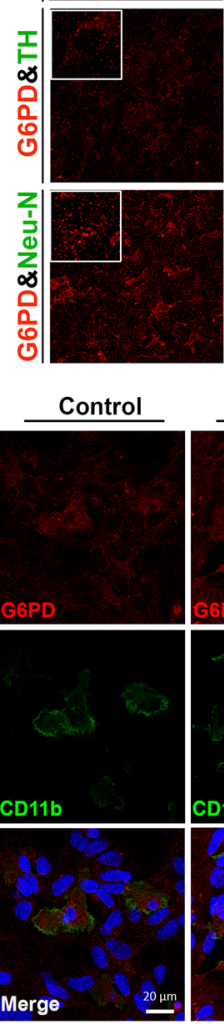
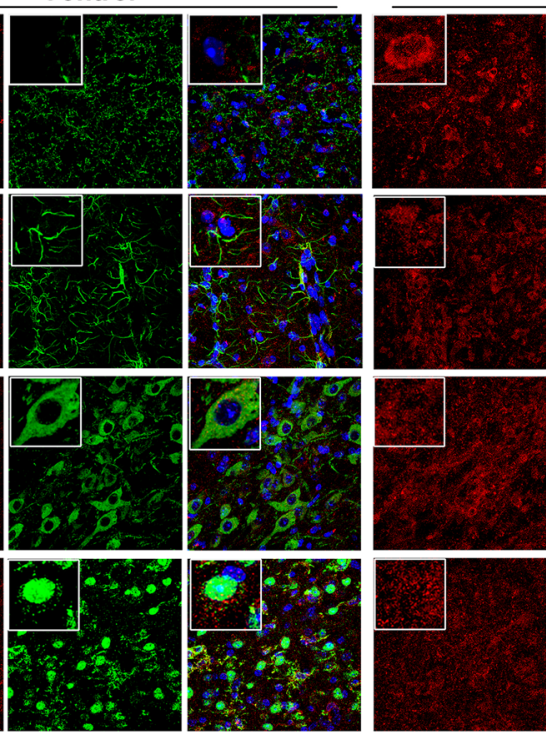

LPS
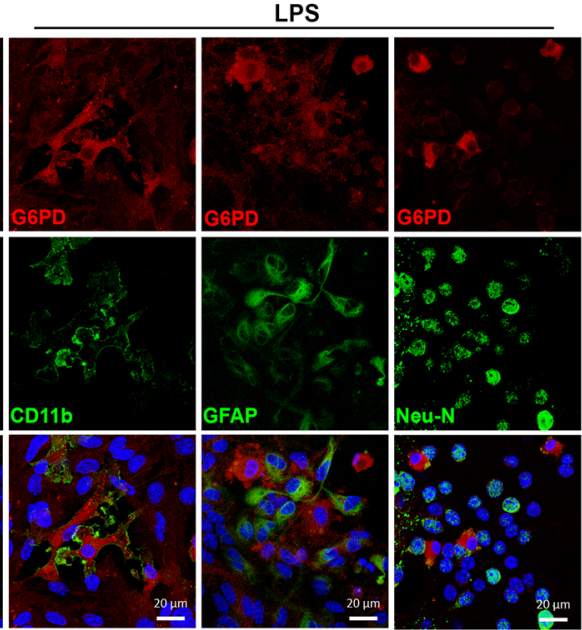

LPS
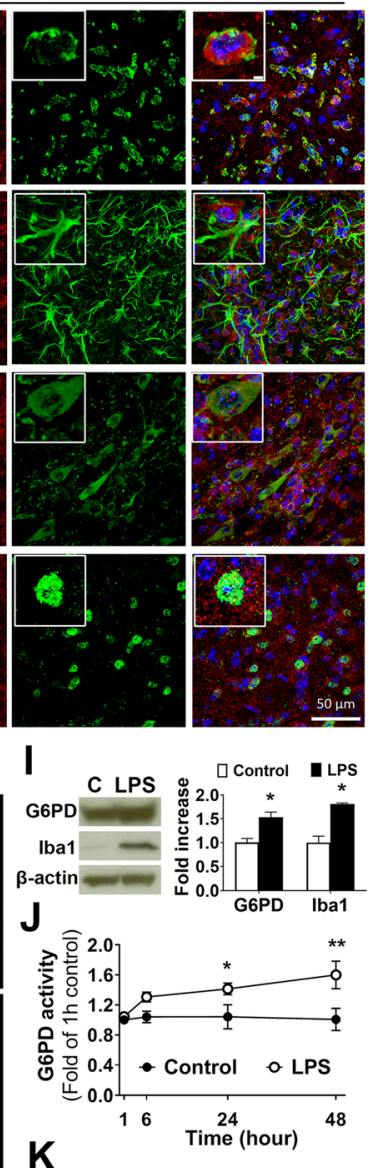

K

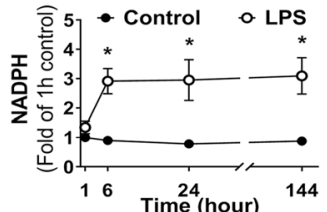

Fig. 1 Increased expression and activity of the PPP in multiple PD models. a One year after an intraperitoneal injection of 5 mg/kg LPS, mouse midbrains displayed increased expression of G6PD, gp9 ${ }^{\text {phox }}$, and Iba1 and decreased expression of TH. $\mathbf{b}$ The ratio of densitometry values of these proteins in a was analyzed and normalized to each responsive control. c The mRNA level of G6PD in the midbrain of mice with an intraperitoneal injection of saline or $5 \mathrm{mg} / \mathrm{kg}$ LPS. $\mathbf{d}$ Double-labeled immunofluorescence of G6PD (red) with microglial marker CD11 b, astroglial marker GFAP, DA neuron maker TH, or neuronal marker Neu-N (green) in mouse SN displayed dramatic G6PD upregulation in microglia at 2 weeks after an intranigral injection of $2 \mu \mathrm{g}$ LPS. Enlarged inserts in the top panel showed increased G6PD staining in microglia. e, $\mathbf{f}$ At 3 or 7 months after daily subcutaneous injection of MPTP ( $15 \mathrm{mg} / \mathrm{kg}$ for 6 days), mouse midbrains exhibited increased expression of G6PD, gp91 phox , and Iba1 and reduced levels of TH. $\mathbf{g}$ One-year-old A53T a-synuclein transgenic mice revealed upregulation of G6PD and gp91 ${ }^{\text {phox }}$ in midbrains compared with age-matched wild-type mice. $\mathbf{h}$ Double-labeled immunofluorescence of G6PD (red) with CD11b, GFAP, or Neu-N (green) in neuron-glia cultures treated with LPS (10 ng/mL) for $48 \mathrm{~h}$ showed occurrence of LPS-induced upregulation of G6PD in activated microglia but not neurons or astroglia. Double-stained images of G6PD and CD11b in vehicle-treated control cultures, neurons, or astroglia, which were negative for CD11b staining and positive for DAPI staining, showed weak G6PD staining. $\mathbf{i}$ Increased expression of G6PD and Iba1 in microglia-enriched cultures upon LPS treatment for $24 \mathrm{~h}$. j, $\mathbf{k}$ Rat mesencephalic neuron-glia cultures treated with LPS showed high activity of G6PD (j) and increased production of $\mathrm{NADPH}(\mathbf{k})$ compared with vehicle-treated cultures. All images are representative of three independent experiments. Results are the mean \pm SEM of three independent experiments $(\mathbf{b}, \mathbf{c}, \mathbf{f}, \mathbf{g}, \mathbf{i}-\mathbf{k}) .{ }^{*} p<0.05$ and ${ }^{* *} p<0.01$ compared with vehicle-treated controls $(\mathbf{b}, \mathbf{c}, \mathbf{f}, \mathbf{i}-\mathbf{k}) .{ }^{*} p<$ 0.05 compared with wild-type littermates $(\mathbf{g})$

Knockdown or pharmacological inhibition of G6PD protected DA neurons from LPS-induced degeneration To explore whether there is a causal relationship between abnormal upregulation of G6PD and DA neurodegeneration, we utilized biological knockdown and pharmacological inhibition to decrease G6PD expression and activity. We prepared reconstituted cultures by adding microglia with 30 -h knockdown of 
G6PD by siRNAs, which caused $45-53 \%$ decreases in G6PD protein levels, onto neuron-astrocyte layer (Fig. 2a). Western blotting results showed that LPS treatment for 6 days led to reduction in $\mathrm{TH}$ protein in reconstituted cultures containing microglia transfected with scramble siRNA (SS) but not G6PD siRNAs (GS1 or GS2). In another word, siRNA-mediated knockdown of microglial G6PD prevented LPS-induced reduction in $\mathrm{TH}$ protein levels (Fig. 2b). Immunocytochemical analysis demonstrated that LPS-induced degeneration of DA neurons, as shown by a significant loss of TH-IR perikaryon and destruction of TH-IR dendrites, was rescued by G6PD knockdown (Fig. 2c, d). Notably, following the LPS treatment in the cultures transfected with scramble siRNA, the number of TH-IR neurons was decreased, and the neurites of the remaining TH-IR neurons became shorter, lighter-stained, or even fragmented. G6PD knockdown protected DA neurons from such destruction (Fig. 2c, d).

We next determined how DA neurodegeneration was affected by inhibition of G6PD activity by 6-AN and DHEA that have been widely used to inhibit G6PD and the PPP to decrease intracellular NADPH levels [2932]. DHEA is a steroid hormone, 6-AN has been reported to induce astroglial toxicity and neurotoxicity in specific nuclei in brainstem and spinal cord [33, 34], and no better G6PD inhibitor is available. We therefore used both 6-AN and DHEA in in vitro studies making the results sound and solid. We first confirmed that both 6-AN $(10 \mu \mathrm{M})$ and DHEA $(100 \mu \mathrm{M})$ blocked LPSelicited increases in G6PD activity and NADPH production in rat mesencephalic neuron-glia cultures at 2 days after LPS treatment (Fig. 2e). LDH and MTT assays detected no cytotoxicity in rat neuron-glia cultures treated with 6-AN $(10 \mu \mathrm{M})$ or DHEA $(100 \mu \mathrm{M})$ for 1 or 7 days (data not shown). Pretreatment of mesencephalic neuron-glia cultures with 6-AN (1-10 $\mu \mathrm{M})$ and DHEA $(10-100 \mu \mathrm{M})$ significantly attenuated LPS-induced degeneration of DA neurons, which was determined by the functional assay of $\left[{ }^{3} \mathrm{H}\right] \mathrm{DA}$ uptake, western blotting assay of $\mathrm{TH}$ protein, and immunocytochemical analysis of TH-IR neurons 7 days after LPS treatment (Fig. 2fj). Collectively, both knockdown of G6PD expression and inhibition of G6PD activity prevented LPS-induced dopaminergic degeneration.

\section{Knockdown and pharmacological inhibition of G6PD attenuated LPS-elicited oxidative stress}

Emerging evidence has indicated that over-activated NOX2, as a major source of oxidative stress under inflammatory condition, plays a pivotal role in chronic neuroinflammation and progressive neurodegeneration [10]. We hypothesized that elevated activity in the PPP increased NADPH production and thereby provided abundant substrate to over-activated NOX2 during neuroinflammation promoting NOX2-derived oxidative stress and subsequent neurodegeneration. To test this hypothesis, we determined whether neuroprotective effects of knockdown and inhibition of G6PD were linked to attenuation of oxidative stress. The results showed that LPS-triggered superoxide release from microglia, measured by SOD-inhibitable reduction of WST-1, was significantly attenuated by siRNA-mediated G6PD knockdown and pharmacological inhibition of G6PD by 6-AN and DHEA (Fig. 3a, b). In addition, pretreatment with 6-AN or DHEA inhibited iROS production in a dose-dependent manner in rat mixed-glia cultures detected by fluorescent probe $\mathrm{CM}-\mathrm{H}_{2}$ DCFDA at $6 \mathrm{~h}$ after LPS treatment (Fig. 3c). Similarly, G6PD knockdown in mouse microglia decreased production of iROS at 2 days after LPS treatment (Fig. 3d, e). Moreover, pretreatment of microglia-enriched cultures for 30 min with 6-AN $(10 \mu \mathrm{M})$ and DHEA $(100 \mu \mathrm{M})$ significantly attenuated LPS-induced production of iROS and 4-HNE (a major end product of lipid peroxidation and a biomarker for oxidative stress) detected at 2 days after LPS treatment (Fig. 3f, g). LDH and MTT assays detected no cytotoxicity in mouse microglia-enriched cultures treated with 6-AN $(10 \mu \mathrm{M})$ or DHEA $(100 \mu \mathrm{M})$ for 1 or 2 days (data not shown). Together, suppression of G6PD activity reduced LPS-elicited oxidative stress.

\section{Inhibition of PPP activity ameliorated inflammatory response}

Previous studies have shown that peripheral blood mononuclear cells from human subjects with G6PDdeficiency exhibit reduced secretion of inflammatory cytokines such as TNF $\alpha$ and IL-1 $\beta$ [35]. Previous findings including ours indicate that oxidative stress and inflammatory response interact and promote each other [10]. Next, we further investigated whether inhibition of PPP ameliorated LPS-induced inflammatory response and microglial activation. First, detection of knockdown efficiency of G6PD siRNAs in mouse microglia by Western blotting assay showed that the level of G6PD was decreased by 53\% after 30-h knockdown by siRNAs (Fig. 4a). Then, at $30 \mathrm{~h}$ after transfection with scramble siRNA and G6PD siRNAs, mouse microglia-enriched cultures were challenged with vehicle or LPS and the microglia inflammatory response was evaluated. Both siRNAs of G6PD decreased LPS-induced release of proinflammatory cytokine TNF $\alpha$ (Fig. 4b). Morphological evaluation showed that LPS-triggered increases in the number, cell size, and expression of Iba-1 (a specific marker of microglia/macrophages) in microglia were significantly mitigated by G6PD knockdown (Fig. 4c, d). Western blotting assay indicated that while LPS- 


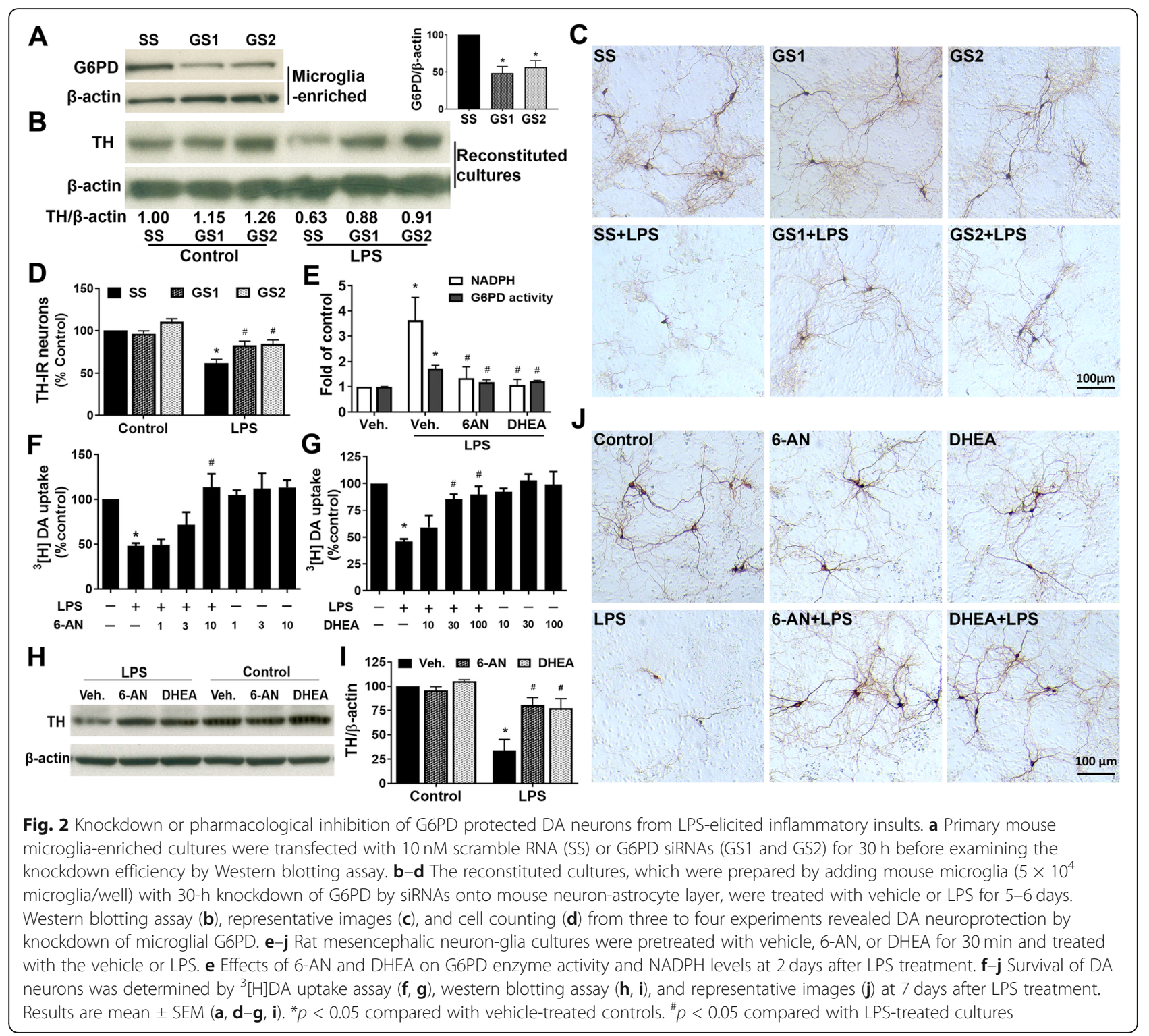

induced G6PD upregulation was prevented by G6PD siRNAs; in microglia with G6PD knockdown, LPS did not induce Iba1 upregulation (Fig. 4e, f). No cytotoxicity was detected in the cell viability assays (LDH and MTT assays) in microglia-enriched cultures transfected with scramble siRNA and two siRNAs of G6PD for $30 \mathrm{~h}$, which ruled out the possibility that the observed anti-inflammatory effects of G6PD knockdown resulted from cell damage/death caused by transfection process (data not shown). Similarly, LPS-induced inflammatory responses including TNF $\alpha$ release, morphological changes, and Iba1 upregulation in microglia in rat neuron-glia cultures were attenuated by pretreatment with 6-AN $(10 \mu \mathrm{M})$ and DHEA $(100 \mu \mathrm{M})$ for $30 \mathrm{~min}$ prior to the addition of LPS.
Notably, neither 6-AN nor DHEA affected microglial survive or morphology (Fig. 4g-k). Thus, knockdown of G6PD and inhibition of G6PD activity ameliorated inflammatory response.

\section{Knockdown and inhibition of G6PD suppressed NF-KB activation}

It is well known that NF- $\mathrm{BB}$ participates in cellular responses to stimuli such as stress, cytokines, and free radicals and plays a key role in regulating immune responses. To examine whether suppression of inflammatory responses of microglia by G6PD knockdown or inhibition was mediated by inhibition of NF- $\mathrm{KB}$ activation. Activation of the NF- $\mathrm{kB}$ pathway, as shown by the phosphorylation and nuclear translocation of p65, was 


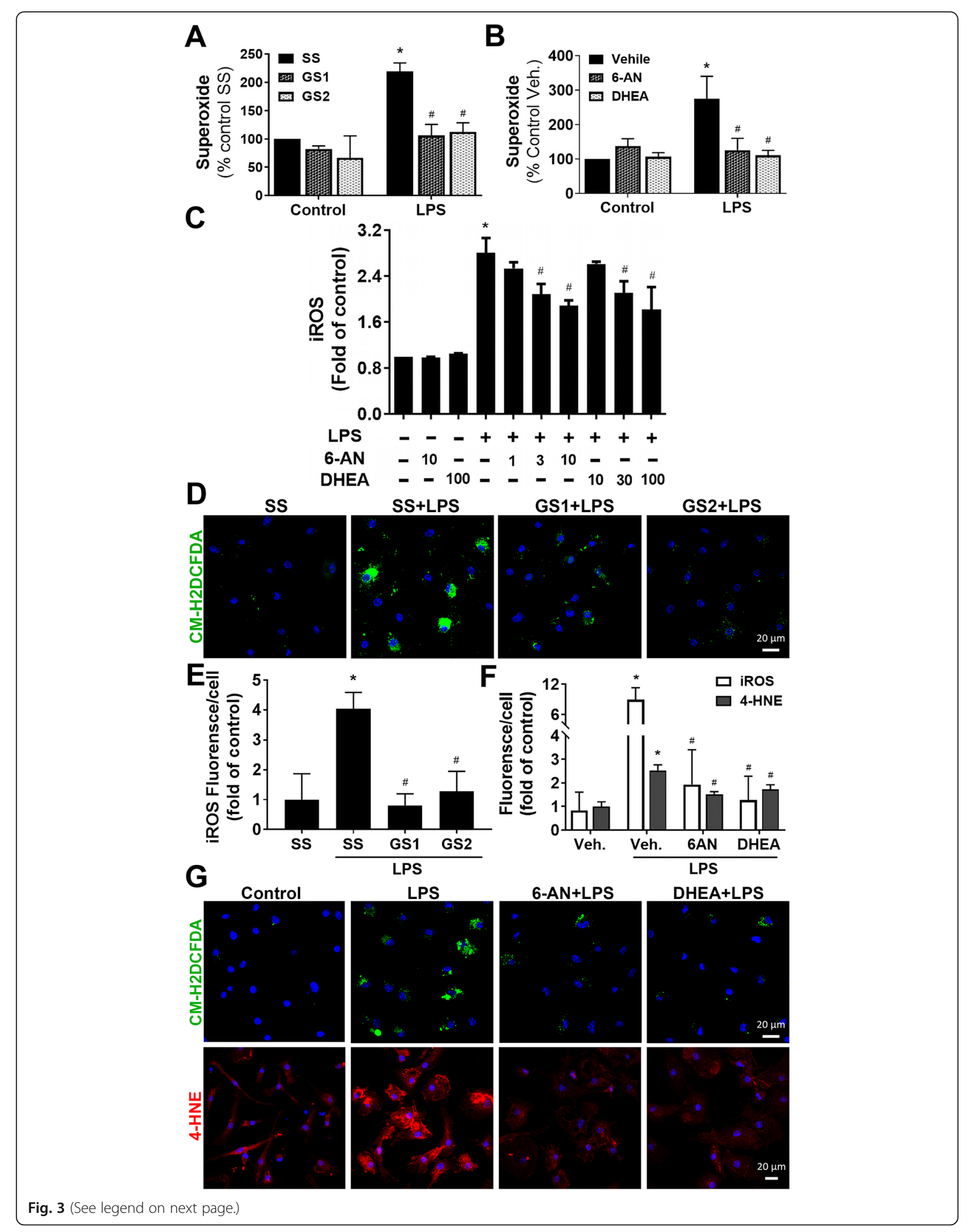


(See figure on previous page.)

Fig. 3 Knockdown or pharmacological inhibition of G6PD attenuated LPS-elicited ROS production. a Microglia-enriched cultures grown in culture chambers were transfected with $10 \mathrm{nM}$ scramble siRNA (SS) or G6PD siRNAs (GS1 and GS2), and $30 \mathrm{~h}$ later, the cultures were treated with LPS. Superoxide production in microglia-enriched cultures was measured by SOD-inhibitable WST-1 reduction. b G6PD inhibitors 6-AN and DHEA attenuated superoxide production in microglia-enriched cultures. c Rat mixed-glia cultures grown in black 96-well plates were pretreated with vehicle, 6-AN, or DHEA at the indicated concentration for 30 min prior to the addition of LPS in phenol red-free medium. Fluorescent probe $\mathrm{H}_{2}$ DCFDA was used to detect inhibition of 6-AN or DHEA on iROS production at $6 \mathrm{~h}$ after LPS addition. $\mathbf{d}$, e At 2 days after LPS treatment, iROS were detected by fluorescent probe $\mathrm{CM}-\mathrm{H}_{2} \mathrm{DCFDA}(\mathbf{d})$ and quantification of the fluorescent intensity (e). $\mathbf{f}, \mathbf{g}$ At 2 days after LPS treatment, CM$\mathrm{H}_{2}$ DCFDA (green) or anti-4-HNE antibody (red) detected decreases in oxidative stress in microglia-enriched cultures pretreated with $10 \mu \mathrm{M}$ 6-AN and $100 \mu \mathrm{M}$ DHEA. Results are mean \pm SEM of three to four experiments performed in triplicate $(\mathbf{a}-\mathbf{c}, \mathbf{e}, \mathbf{f}) .{ }^{*} p<0.05$ compared with vehicletreated controls. ${ }^{\#} p<0.05$ compared with LPS-treated cultures

observed after LPS stimulus in microglia-enriched cultures challenged with LPS for 15, 30, and $60 \mathrm{~min}$ (Fig 5a). Pretreatment of microglia with 6 -AN $(10 \mu \mathrm{M})$ or DHEA $(100 \mu \mathrm{M})$ for $30 \mathrm{~min}$ attenuated LPS-induced phosphorylation and nuclear translocation of p65 (Fig. 5b-d). Similarly, phosphorylation and nuclear translocation of p65 in LPS-treated microglia-enriched cultures were also prevented by G6PD knockdown (Fig. 5e-g).
G6PD inhibition by 6-AN injection attenuated LPSinduced oxidative stress, inflammatory response, dopaminergic neurodegeneration, and locomotor impairment

As described above, G6PD knockdown and inhibition attenuated LPS-induced oxidative stress, inflammatory response, and loss of DA neurons in cell culture systems. We next performed intranigral injection of saline or 6AN $(20 \mathrm{nmol})$ with LPS $(2 \mu \mathrm{g})$ into C57BL/6 J mice.

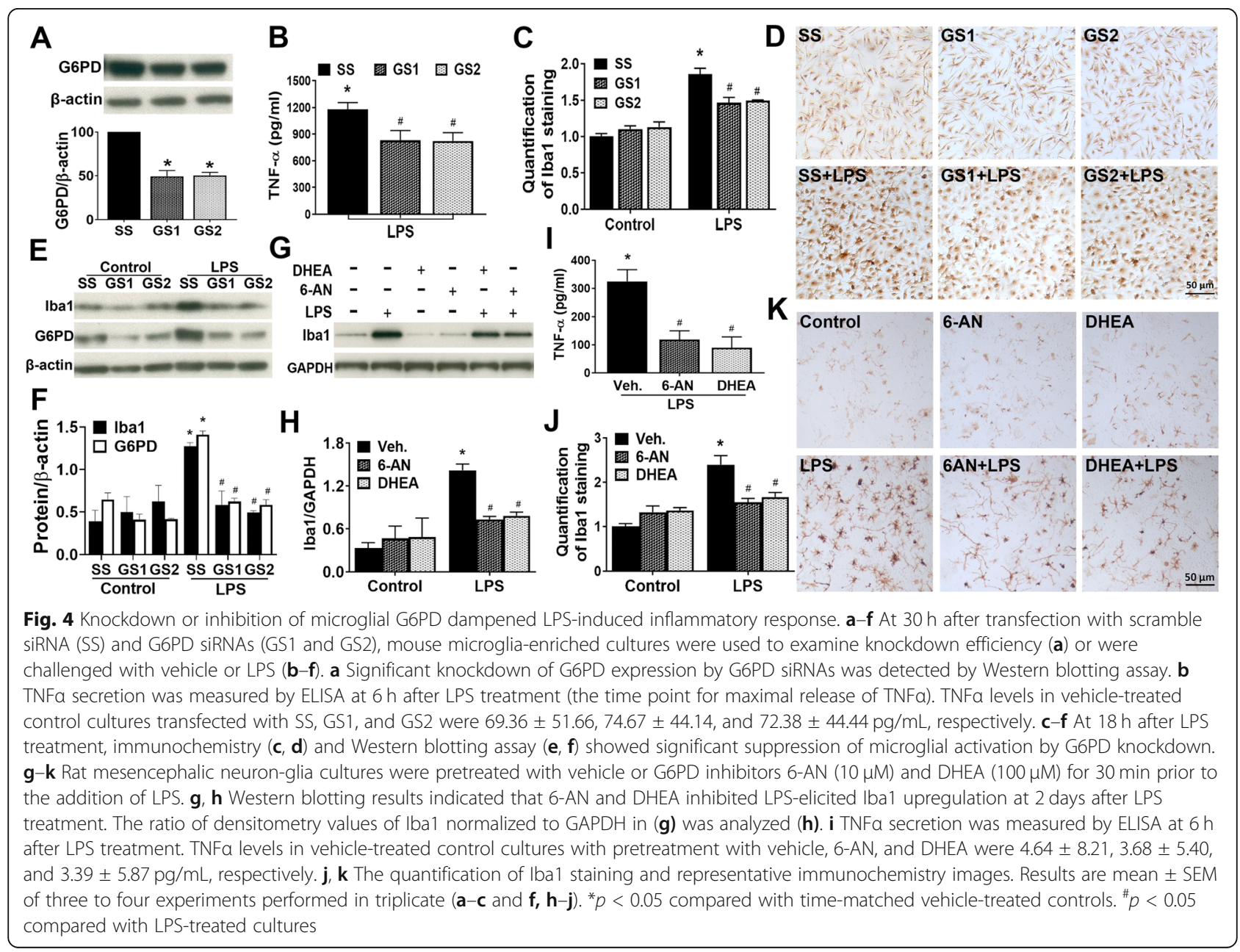




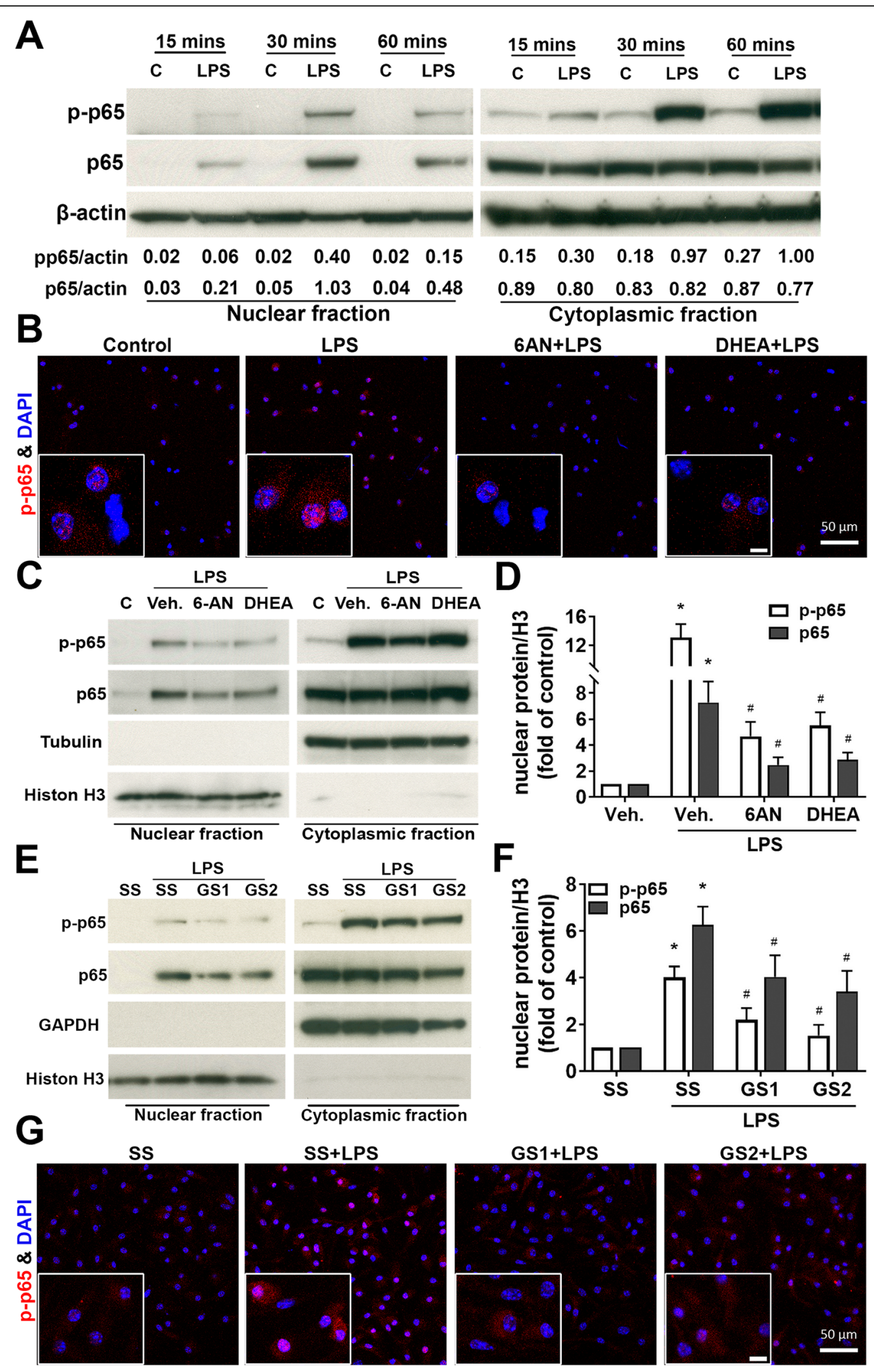

Fig. 5 (See legend on next page.) 


\section{(See figure on previous page.)}

Fig. 5 Inhibition or knockdown of G6PD suppressed NF-KB activation. a Microglia-enriched cultures were challenged by LPS for 15, 30, and 60 min. The activation of NF-KB pathway, as shown by phosphorylation and nuclear translocation of p65, was observed after LPS treatment. $\mathbf{b}-\mathbf{d}$ Microglia-enriched cultures were pretreated with vehicle, 6-AN $(10 \mu \mathrm{M})$, or DHEA $(100 \mu \mathrm{M})$ for 30 min prior to the addition of LPS. $\mathbf{b}$ Immunofluorescence was performed with phosphorylated p65 (red) 30 min after LPS treatment. c, d Nuclear translocation of p-p65 and p65 was examined after the cultures were treated with 6-AN or DHEA. e- $\mathbf{g}$ At $30 \mathrm{~h}$ after transfection with scramble siRNA (SS) and G6PD siRNAs (GS1 and GS2), mouse microglia-enriched cultures were challenged with vehicle or LPS. Nuclear and cytoplasmic proteins were examined at 30 min after LPS treatment to determine the effect of reduced G6PD on NF-KB activation. Results are mean \pm SEM of two to three experiments performed in triplicate $(\mathbf{a}, \mathbf{d}, \mathbf{f}) .{ }^{*} p<0.05$ compared with vehicle-treated controls. ${ }^{*} p<0.05$ compared with LPS-treated cultures

Considering the nature of DHEA as a steroid hormone and the lack of cell toxicity of 6-AN at doses showing anti-inflammation and neuroprotection in our in vitro studies, we chose to use 6-AN to do the in vivo experiment. At 6 weeks after the injection, oxidative stress, microglial activation, and DA neuron damages were evaluated by immunofluorescence. 3-Nitrotyrosine (3NT) is thought to be a relatively specific marker of oxidative damage [36] and has high correlation to neurodegenerative diseases [37]. Injection of 6-AN apparently mitigated LPS-induced oxidative stress in the SN (Fig. $6 \mathrm{a}, \mathrm{b})$. Increased 3-NT staining was mainly located in microglia and remaining DA neurons (Fig. 6a). Consistent with in vitro findings, in vivo studies also showed that LPS-induced microglial activation in the SN, as shown by CD11b and Iba1 upregulation and switch from ramified resting morphology to activated morphology with enlarged and irregular cell body, was dampened by 6-AN injection (Fig. 6a, c-e, g, h). In addition, nigral DA neurons were protected from LPS-elicited degeneration by 6-AN, as shown by improvement in the number of $\mathrm{TH}-\mathrm{IR}$ neurons, the expression of $\mathrm{TH}$, and the integrity of TH-IR fibers of the remaining TH-IR neurons in mice co-injected with 6-AN and LPS compared with mice injected with LPS alone (Fig. 6a, c, f, g, i). Moreover, impairment in locomotor activity of mice with LPS injection evaluated by the rotarod behavior test was rescued by 6 -AN injection (Fig. 6j). In short, suppression of G6PD activation by intranigral injection of 6-AN attenuated LPS-elicited oxidative stress, microglial activation, dopaminergic neurodegeneration, and locomotor impairment.

\section{Discussion}

The present study has demonstrated that aberrant upregulation of G6PD in microglia exacerbated LPSinduced chronic neurodegeneration through amplifying cellular oxidative stress and inflammatory response. Elevated expression and activity of G6PD were consistently observed in multiple in vitro and in vivo PD models. Knockdown of microglial G6PD and inhibition of G6PD activity by 6-AN and DHEA prevented DA neurons from LPS-induced chronic degeneration. Further mechanistic studies indicated that increased expression and activity of G6PD enhanced NADPH production thereby amplifying LPS-elicited NOX2-derived oxidative stress and NF- $\mathrm{kB}$ activation in microglia; these effects were attenuated by knockdown of microglial G6PD and inhibition of G6PD by 6-AN and DHEA. Our findings indicated that G6PD-mediated PPP dysfunction and elevated NADPH production in microglia led to increased production of NOX2-derived ROS amplifying microglial activation and neurodegeneration. Thus, this study has provided the first evidence demonstrating that metabolic disruption in the PPP played a pivotal role in inflammation-induced dopaminergic neurodegeneration.

Studies in animal models and patients have shown glucose metabolism disruption in PD [2, 13, 14]. For instance, PD patients exhibit a decrease in glucose metabolism and abnormal elevation in levels of lactate/ pyruvate [38-40]. It is of special interest that several recent studies have revealed dysregulation of the PPP in PD $[2,13]$. Transgenic mice with specific overexpression of G6PD in DA neurons are resistant to dopaminergic neurotoxin MPTP [41]. PD-related pesticide paraquat, a superoxide-producing oxidant, increases PPP metabolites (e.g. glucose-6-phosphate and fructose-6-phosphate) and G6PD expression in dopaminergic cell lines, inducing oxidative stress and neurotoxicity that is enhanced by $\alpha$ synuclein overexpression [42, 43]. Moreover, a postmortem study of PD brains has revealed an increase in $\mathrm{NADPH}$ production in the putamen (a brain region affected in PD) but not in the cortex or the cerebellum (unaffected or least affected brain regions in PD) of latestage cases; unexpectedly, the putamen of early-stage PD and the cerebellum of early- and late-stage PD display a reduction in G6PD [13]. Here, based on regional patterns of Lewy body pathology, PD cases have been grouped into early- and late-stage in which Lewy body pathology is confined to the brainstem and spreads to the limbic and neocortical areas, respectively. However, whether Lewy body pathology accurately reflects the clinical severity and disease progression course of PD is still under debate. In addition, neither NADPH nor G6PD in the SN (the major disease region in PD) has been examined. Earlier measurement of G6PD in red blood cells in PD patients has yielded conflicting results claiming reduced or unaltered activity [20, 21]. The present study detected sustained 


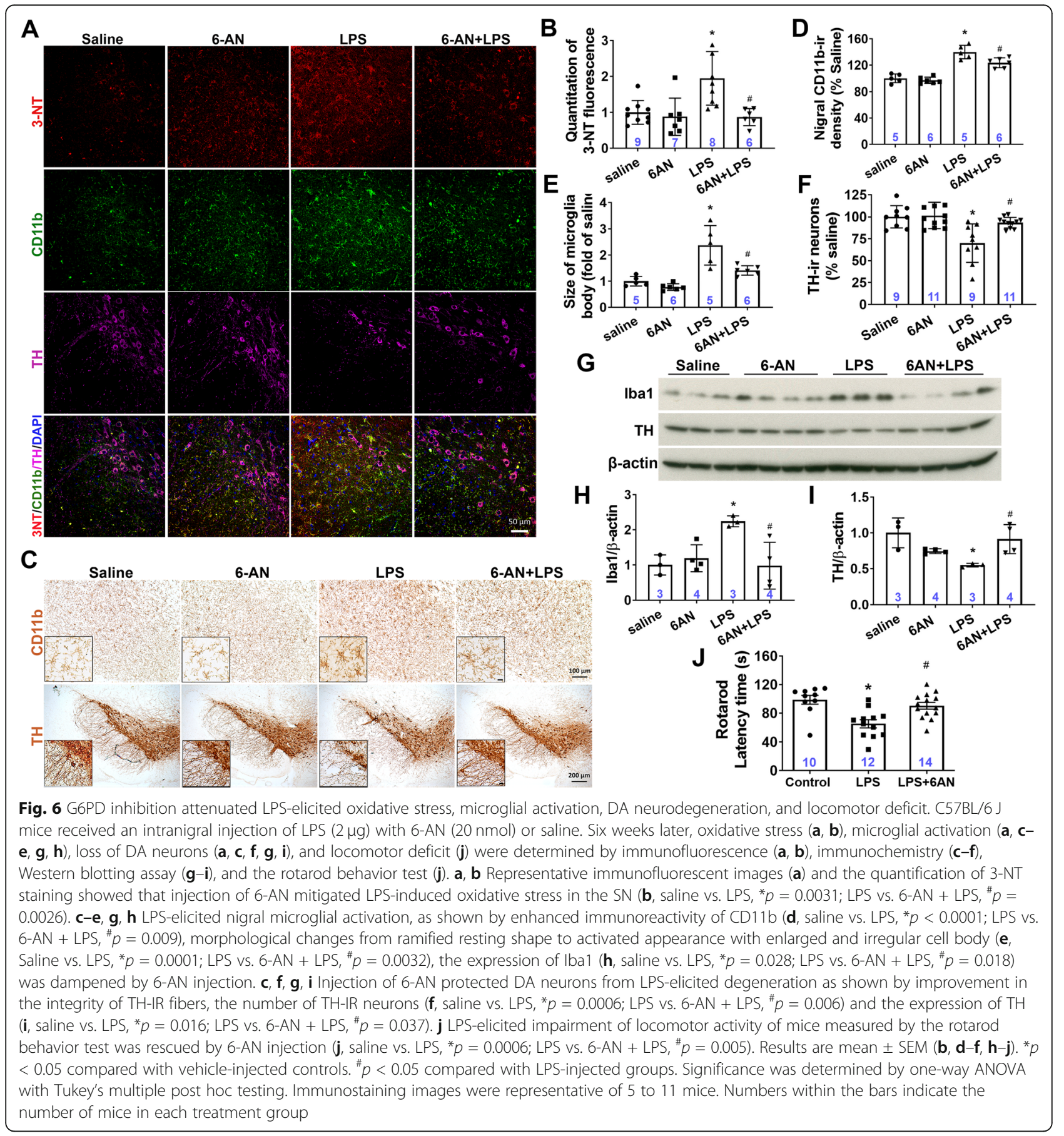

elevation in expression and activity of G6PD in LPStreated mesencephalic neuron-glia cultures (an in vitro PD model) and in the SN of four in vivo PD models (Fig. 1). In addition to difference in sample size, examined materials (RBC, leukocyte, neuronal cell lines, human/mouse brain tissues), measurement methods/sensitivity, and disease stage/severity, dynamic changes in the activity of G6PD in different stages of PD might also contribute to the significant heterogeneity among studies [13, 20, 21].
More importantly, inhibition of G6PD and knockdown of microglial G6PD significantly attenuated LPS-elicited dopaminergic neurodegeneration in both in vitro and in vivo models of PD (Figs. 2 and 6). Our findings provided experimental evidence to indicate that G6PD upregulation contributed to PD pathogenesis.

As one of the three major pathways for the body to generate reducing molecules, the PPP produces approximately $60 \%$ of $\mathrm{NADPH}$ in humans [44]. NADPH 
provides the reducing equivalents for biosynthetic/anabolic reactions (e.g. lipid/cholesterol synthesis and fatty acid chain elongation) and oxidation-reduction reactions. NADPH serves as a cofactor of glutathione reductase in generating the reduced glutathione (GSH), one of the most important antioxidants [45-47]. In neurons, $\mathrm{NADPH}$ is used as a cofactor for synthesis of fatty acids and myelin, for neurotransmitter turnover, and for redox homeostasis maintenance. NADPH is also required for free radical production by NOX2 and nitric oxide synthase [48]. Thus, NADPH is a central component of both anti- and pro-oxidant processes. The PPP plays an important role in a variety of seemingly diverse human diseases with redox dysregulation as a common denominator, such as hemolytic anemia, autoimmune diseases (e.g. lupus and multiple sclerosis), and male infertility [16]. In some peripheral pathological conditions (e.g. heart failure, atherosclerosis, and obesity), increased activity of G6PD promotes cellular ROS production and pro-inflammatory signaling through increased availability of NADPH to ROS-producing enzymes [32, 49-52]. Furthermore, G6PD upregulation in the heart, liver, and pancreatic $\beta$ cells of obese and diabetic animals leads to functional defects in the respective tissue by increasing oxidative stress [53-55]. Notably, the $\mathrm{SN}$ of patients with PD and mouse models of PD displays upregulation of NOX2 (Fig. 1a, b, e-g) [12]. Over-activated NOX2 is a major source of oxidative stress under inflammatory condition and has been implicated as a novel therapeutic target for neurodegenerative diseases [10]. Microglia with elevated expression and activity of G6PD produced excessive NADPH (Fig. 1) and provided abundant substrate to over-activated NOX2 promoting oxidative stress (Fig. 7).

It is of special interest that G6PD displays high activity in immune cells and participates in the modulation of oxidative stress and inflammatory reactions. An abnormal increase of G6PD in macrophages promotes oxidative stress and inflammatory responses in the adipose tissue of obese mice [56]. G6PD deficiency renders macrophages resistance to LPS stimulation and attenuates insulin resistance in obesity mice through inhibition of adipose tissue inflammation [57]. It has been reported that activated microglia stimulated by LPS with or without interferon $\gamma$ switch their metabolism from oxidative phosphorylation to glycolysis that also enhances carbon flux to the PPP [58-61]. Knockdown of microglial G6PD or pharmacological inhibition of G6PD activity attenuated LPS-elicited inflammatory responses and oxidative stress (Figs. 3, 4, 5, 6, and 7). Thus, G6PD upregulation and PPP activity elevation in innate immune cells play important roles in chronic glucose metabolism disruption, oxidative stress, and inflammatory responses in both peripheral tissues and the CNS.

Cooperation among different cells in the CNS and optimal balance in the PPP and glycolysis in glucose metabolism must be extremely critical for bioenergetics, redox balance, immune homeostasis, and neuronal

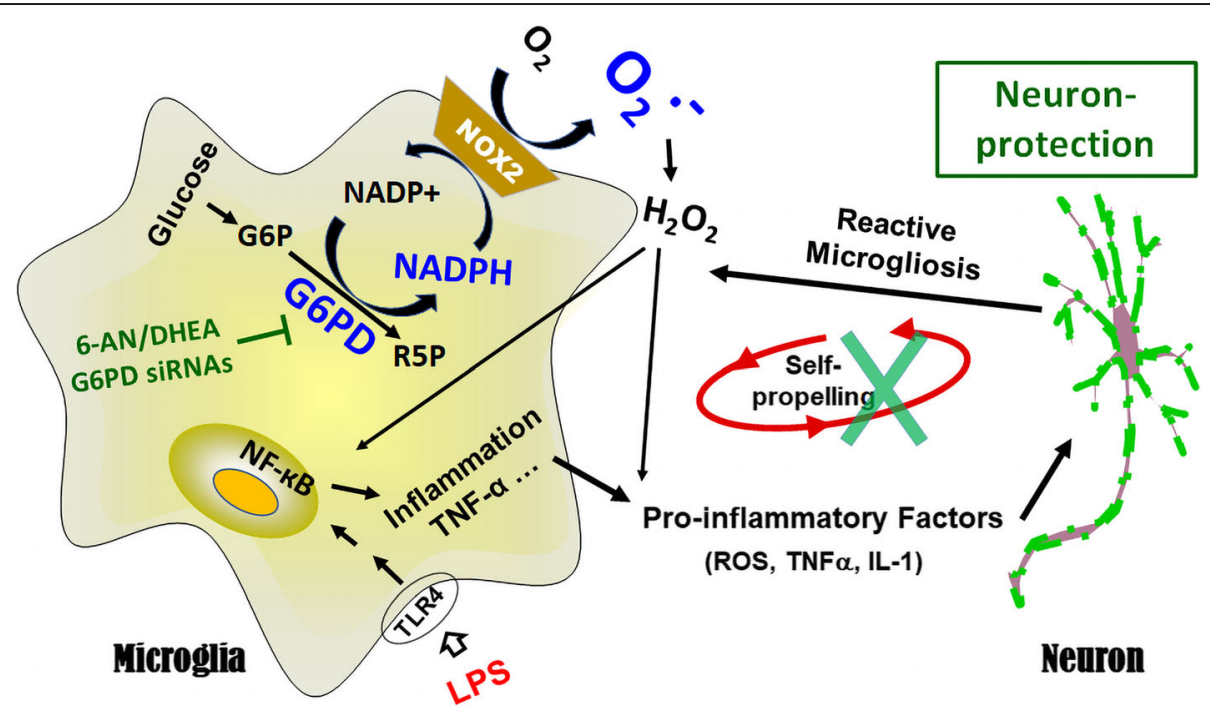

Fig. 7. Exacerbation between G6PD dysfunction and neuroinflammation mediated chronic neurodegeneration. The present study elucidated a pathophysiological role of microglial G6PD in PD neurodegeneration. LPS-induced aberrant upregulation of G6PD promoted cellular oxidative stress and NF-KB activation through increasing NADPH availability to NADPH oxidase. Pharmacological inhibition of G6PD activity or biological knockdown of G6PD attenuated oxidative stress, ameliorated inflammatory response of microglia, and prevented DA neurons from LPS-induced chronic degeneration. G6P, glucose-6-phosphate; G6PD, glucose-6-phosphate dehydrogenase; PPP, pentose phosphate pathway; NADPH, nicotinamideadenine-dinucleotide phosphate; NADP ${ }^{+}$, oxidized form of NADPH; R5P, ribose-5-phosphate; LPS: Lipopolysaccharide; 6-AN, 6aminonicotinamide; DHEA, dehydroepiandrosterone; $\mathrm{O}_{2}{ }^{-}$, superoxide; $\mathrm{H}_{2} \mathrm{O}_{2}$, hydrogen peroxide; $\mathrm{ROS}$, reactive oxygen species 
survival and should become priority in investigating glucose metabolism dysregulation in brain diseases. Several recent studies have explored cooperation between astrocytes and neurons in glucose metabolism [19, 62, 63]. Disruption of glycolysis in astrocytes and oligodendrocytes triggers axon damage and neurodegeneration [64, 65]. Our findings suggest two-way traffic between metabolic reprogramming and innate immune response in PD pathogenesis. LPS-elicited neuroinflammation triggered abnormal elevation of G6PD expression and activity in microglia, which in turn exacerbated neuroinflammation thereby inducing dopaminergic neurodegeneration. Oxidative stress is the shared predominant mediator during this pathogenic process. Indeed, it has recently been proposed that metabolic-inflammatory axis plays important roles in brain aging and neurodegeneration [22].

Multiple lines of evidence including ours from the present study reveal important contribution of G6PD/ PPP dysfunction and consequent oxidative stress to PD pathogenesis and suggest that modulation of G6PD activity might be beneficial for PD treatment. However, caution is needed on targeting G6PD and the PPP as a therapeutic intervention, because neurons greatly rely on the PPP to generate NADPH and its downstream antioxidant GSH to maintain their redox homeostasis, integrity, and normal function. Unlike most other cell types, neurons preferentially metabolize glucose via the PPP because constant ubiquitination and proteasomal degradation of phosphofructokinase $\mathrm{B} 3$, the rate-limiting enzyme and master regulator of glycolysis [19]. G6PD deficiency, an X-linked recessive genetic disorder, is the most common enzymopathy affecting approximately 400 million people worldwide. The most common clinical manifestation associated with G6PD deficiency is hemolytic anemia [66]. Acute hemolysis associated with G6PD deficiency usually is short-lived and self-limited. G6PD deficiency is a manageable disease that requires strict avoidance of oxidative stressor in the form of infection, oxidative drugs, or foods (e.g. fava beans). These features of G6PD suggest that it is feasible to treat PD through dynamic modulating G6PD activity and to avoid compromising RBCs, neurons, the immune system, and other physiological function.

\section{Conclusions}

Overall, our data demonstrated, for the first time, that G6PD and the PPP played a pivotal role in LPS-induced degeneration of DA neurons and locomotor impairment. Metabolic disruption in the PPP and neuroinflammation exacerbated each other inducing PD-like neurodegeneration and locomotor impairment. Results of this study shed light on metabolic-inflammatory mechanisms of PD pathogenesis and suggest that modulation of activity of G6PD and NOX2 may become potential therapeutic interventions in PD.

\section{Supplementary information}

Supplementary information accompanies this paper at https://doi.org/10. 1186/s12974-019-1659-1.

Additional file 1: Figure S1. The pattern of G6PD expression in microglia-containing cultures. (A) Immunocytochemical staining for G6PD on neuron-glia cultures (left panel) and microglia-enriched cultures (middle panel) and double-labeled immunofluorescent staining on microgliaenriched cultures (right panel) detected a significant increase in the level of G6PD protein at 7 days and $24 \mathrm{~h}$ after LPS treatment for neuron-glia cultures and microglia-enriched cultures respectively. (B) Densitometric measurements of G6PD immunoreactivity in (A).

\section{Abbreviations}

3-NT: 3-Nitrotyrosine; 4-HNE : 4-Hydroxynonenal; 6-AN: 6-Aminonicotinamide; $\mathrm{CM}-\mathrm{H}_{2} \mathrm{DCFDA}$ : Chloromethyl-29, 79-dichlorodihydrofluorescein diacetate; CNS: Central nervous system; DA: Dopamine;

DHEA: Dehydroepiandrosterone; FBS: Fetal bovine serum; G6PD: Glucose-6phosphate dehydrogenase; GAPDH: Glyceraldehyde 3-phosphate dehydrogenase; GFAP: Glial fibrillary acidic protein; GS1/GS2: G6PD siRNAs; GSH: Reduced glutathione; HLA: Human leukocyte antigen; HS: Horse serum; Iba1: Ionized calcium-binding adaptor molecule-1; IL-1 $\beta$ : Interleukin 1 beta; iROS: Intracellular reactive oxygen species; LDH: Lactate dehydrogenase; LME: Leucine-leucine methyl ester; LPS: Lipopolysaccharide; MEM: Minimum essential medium; MPTP: 1-Methyl-4-phenyl-1,2,3,6-tetrahydropyridine; MTT: Thiazolyl blue tetrazolium bromide; NADPH: Nicotinamide adenine dinucleotide phosphate; Neu-N: Neuron-specific nuclear protein; NFkB: Nuclear factor kappa B; NOX2: NADPH oxidase; PD: Parkinson's disease; PPP: Pentose phosphate pathway; ROS: Reactive oxygen species; SN: Substantia nigra; SOD: Superoxide dismutase; SS: Scramble siRNA; THIR: Tyrosine hydroxylase-immunoreactive; TNFa: Tumor necrosis factor-a; WST-1: Water-soluble tetrazolium salt 1

\section{Acknowledgements}

We thank Anthony Lockhart, J. Tucker, Eli Ney, and Belinda Wilson for mouse colony maintenance, assistance in confocal imaging collection, immunohistochemistry images collection, and laboratory technical support, respectively.

\section{Authors' contributions}

DT and H-MG wrote the paper; J-SH edited the paper; DT, H-MG, and J-SH designed research; DT, YG, RY, and TG performed research; DT, YG, RY, and H-MG analyzed data. All authors read and approved the final manuscript.

\section{Funding}

This work was supported by the National Natural Science Foundation of China Grants 31471006 and 21577004, the national high technology research and development program of China Grants 2014AA021601, National Basic Research Program of China Grants 2015BAI08B02, Fundamental Research Funds for the Central Universities Grants 14913101 and 1480601101, Priority Academic Program Development of Jiangsu Higher Education Institutions, and the award to high-level innovative and entrepreneurial talents of Jiangsu Province of China to Hui-Ming Gao. This work was supported in part by the Intramural Research Program of the NIH/NIEHS (ES090082-22).

\section{Availability of data and materials}

The datasets used and/or analyzed during the current study are available from the corresponding author on reasonable request.

Ethics approval and consent to participate Not applicable

Consent for publication

Not applicable 


\section{Competing interests}

The authors declare that they have no competing interests.

\section{Received: 21 August 2019 Accepted: 26 November 2019} Published online: 05 December 2019

\section{References}

1. Olanow CW, Tatton WG. Etiology and pathogenesis of Parkinson's disease. Annu Rev Neurosci. 1999;22:123-44.

2. Camandola S, Mattson MP. Brain metabolism in health, aging, and neurodegeneration. EMBO J. 2017:36:1474-92.

3. Ransohoff RM. How neuroinflammation contributes to neurodegeneration. Science. 2016;353:777-83.

4. Glass CK, Saijo K, Winner B, Marchetto MC, Gage FH. Mechanisms underlying inflammation in neurodegeneration. Cell. 2010;140:918-34.

5. Gao HM, Hong JS. Why neurodegenerative diseases are progressive: uncontrolled inflammation drives disease progression. Trends Immunol. 2008;29:357-65.

6. Wahner AD, Sinsheimer JS, Bronstein JM, Ritz B. Inflammatory cytokine gene polymorphisms and increased risk of Parkinson disease. Arch Neurol. 2007. 64:836-40

7. Hamza TH, Zabetian CP, Tenesa A, Laederach A, Montimurro J, Yearout D, Kay DM, Doheny KF, Paschall J, Pugh E, et al. Common genetic variation in the HLA region is associated with late-onset sporadic Parkinson's disease. Nat Genet. 2010;42:781-5.

8. McGeer PL, Schwab C, Parent A, Doudet D. Presence of reactive microglia in monkey substantia nigra years after 1-methyl-4-phenyl-1,2,3,6tetrahydropyridine administration. Ann Neurol. 2003:54:599-604.

9. Block ML, Zecca L, Hong JS. Microglia-mediated neurotoxicity: uncovering the molecular mechanisms. Nat Rev Neurosci. 2007:8:57-69.

10. Gao HM, Zhou H, Hong JS. NADPH oxidases: novel therapeutic targets for neurodegenerative diseases. Trends Pharmacol Sci. 2012;33:295-303.

11. Zhang F, Shi JS, Zhou H, Wilson B, Hong JS, Gao HM. Resveratrol protects dopamine neurons against lipopolysaccharide-induced neurotoxicity through its anti-inflammatory actions. Mol Pharmacol. 2010;78:466-77.

12. Wu DC, Teismann P, Tieu K, Vila M, Jackson-Lewis $V$, Ischiropoulos $H$, Przedborski S. NADPH oxidase mediates oxidative stress in the 1-methyl-4phenyl-1,2,3,6-tetrahydropyridine model of Parkinson's disease. Proc Natl Acad Sci U S A. 2003;100:6145-50

13. Dunn L, Allen GF, Mamais A, Ling H, Li A, Duberley KE, Hargreaves IP, Pope $\mathrm{S}$, Holton $\mathrm{J}$, Lees $\mathrm{A}$, et al. Dysregulation of glucose metabolism is an early event in sporadic Parkinson's disease. Neurobiol Aging. 2014;35:1111-5.

14. Backes $H$, Walberer $M$, Ladwig A, Rueger MA, Neumaier B, Endepols $H$, Hoehn M, Fink GR, Schroeter M, Graf R. Glucose consumption of inflammatory cells masks metabolic deficits in the brain. Neuroimage. 2016; 128:54-62.

15. Anandhan A, Jacome MS, Lei S, Hernandez-Franco P, Pappa A, Panayiotidis Ml, Powers R, Franco R. Metabolic dysfunction in Parkinson's disease: bioenergetics, redox homeostasis and central carbon metabolism. Brain Res Bull. 2017:133:12-30.

16. Perl A, Hanczko R, Telarico T, Oaks Z, Landas S. Oxidative stress, inflammation and carcinogenesis are controlled through the pentose phosphate pathway by transaldolase. Trends Mol Med. 2011;17:395-403.

17. Battistuzzi G, D'Urso M, Toniolo D, Persico GM, Luzzatto L. Tissue-specific levels of human glucose-6-phosphate dehydrogenase correlate with methylation of specific sites at the 3' end of the gene. Proc Natl Acad Sci U S A. 1985;82:1465-9.

18. D'Urso M, Mareni C, Toniolo D, Piscopo M, Schlessinger D, Luzzatto L. Regulation of glucose 6-phosphate dehydrogenase expression in $\mathrm{CHO}$ human fibroblast somatic cell hybrids. Somatic Cell Genet. 1983:9:429-43.

19. Herrero-Mendez A, Almeida A, Fernandez E, Maestre C, Moncada S, Bolanos $J P$. The bioenergetic and antioxidant status of neurons is controlled by continuous degradation of a key glycolytic enzyme by APC/C-Cdh1. Nat Cell Biol. 2009;11:747-52

20. Gao L, Mir P, Diaz-Corrales FJ, Mejias R, Carrillo F, Vime PJ, Diaz-Martin J, Palomino A, Carballo M, Pintado E, et al. Glucose-6-phosphate dehydrogenase activity in Parkinson's disease. J Neurol. 2008;255:1850-1.

21. Abraham S, Soundararajan CC, Vivekanandhan S, Behari M. Erythrocyte antioxidant enzymes in Parkinson's disease. Indian J Med Res. 2005;121:111-5
22. Yin F, Sancheti H, Patil I, Cadenas E. Energy metabolism and inflammation in brain aging and Alzheimer's disease. Free Radic Biol Med. 2016.

23. Zhang W, Wang T, Qin L, Gao HM, Wilson B, Ali SF, Zhang W, Hong JS, Liu B. Neuroprotective effect of dextromethorphan in the MPTP Parkinson's disease model: role of NADPH oxidase. Faseb j. 2004;18:589-91.

24. Gao HM, Kotzbauer PT, Uryu K, Leight S, Trojanowski JQ, Lee VM. Neuroinflammation and oxidation/nitration of alpha-synuclein linked to dopaminergic neurodegeneration. J Neurosci. 2008;28:7687-98.

25. Gao HM, Hong JS, Zhang W, Liu B. Distinct role for microglia in rotenoneinduced degeneration of dopaminergic neurons. J Neurosci. 2002;22:782-90

26. Gao HM, Liu B, Hong JS. Critical role for microglial NADPH oxidase in rotenone-induced degeneration of dopaminergic neurons. J Neurosci. 2003;23:6181-7.

27. Zhou H, Zhang F, Chen SH, Zhang D, Wilson B, Hong JS, Gao HM. Rotenone activates phagocyte NADPH oxidase by binding to its membrane subunit gp91phox. Free Radic Biol Med. 2012;52:303-13.

28. Gaire BP, Lee CH, Sapkota A, Lee SY, Chun J, Cho HJ, Nam TG, Choi JW. Identification of sphingosine 1-phosphate receptor subtype 1 (S1P1) as a pathogenic factor in transient focal cerebral ischemia. Mol Neurobiol. 2018:55:2320-32.

29. Kohler $\mathrm{E}$, Barrach $\mathrm{H}$, Neubert D. Inhibition of NADP dependent oxidoreductases by the 6-aminonicotinamide analogue of NADP. FEBS Lett. 1970;6:225-8.

30. Hofgaard JP, Sigurdardottir KS, Treiman M. Protection by 6aminonicotinamide against oxidative stress in cardiac cells. Pharmacol Res. 2006;54:303-10.

31. Raineri R, Levy HR. On the specificity of steroid interaction with mammary glucose 6-phosphate dehydrogenase. Biochemistry. 1970;9:2233-43.

32. Park J, Choe SS, Choi AH, Kim KH, Yoon MJ, Suganami T, Ogawa Y, Kim JB. Increase in glucose-6-phosphate dehydrogenase in adipocytes stimulates oxidative stress and inflammatory signals. Diabetes. 2006;55:2939-49.

33. Krum JM. Age-dependent susceptibility of CNS glial populations in situ to the antimetabolite 6-aminonicotinamide. Mol Chem Neuropathol. 1995;26:79-94

34. Penkowa M, Camats J, Hadberg H, Quintana A, Rojas S, Giralt M, Molinero A, Campbell IL, Hidalgo J. Astrocyte-targeted expression of interleukin-6 protects the central nervous system during neuroglial degeneration induced by 6-aminonicotinamide. J Neurosci Res. 2003;73:481-96.

35. Sanna F, Bonatesta RR, Frongia B, Uda S, Banni S, Melis MP, Collu M, Madeddu C, Serpe R, Puddu S, et al. Production of inflammatory molecules in peripheral blood mononuclear cells from severely glucose-6-phosphate dehydrogenase-deficient subjects. J Vasc Res. 2007;44:253-63.

36. Ahsan H. 3-Nitrotyrosine: a biomarker of nitrogen free radical species modified proteins in systemic autoimmunogenic conditions. Hum Immunol. 2013;74:1392-9.

37. Jomova K, Vondrakova D, Lawson M, Valko M. Metals, oxidative stress and neurodegenerative disorders. Mol Cell Biochem. 2010;345:91-104.

38. Edison P, Ahmed I, Fan Z, Hinz R, Gelosa G, Ray Chaudhuri K, Walker Z, Turkheimer FE, Brooks DJ. Microglia, amyloid, and glucose metabolism in Parkinson's disease with and without dementia. Neuropsychopharmacology. 2013;38:938-49.

39. Ahmed SS, Santosh W, Kumar S, Christlet HT. Metabolic profiling of Parkinson's disease: evidence of biomarker from gene expression analysis and rapid neural network detection. J Biomed Sci. 2009;16:63.

40. Eberling JL, Richardson BC, Reed BR, Wolfe N, Jagust WJ. Cortical glucose metabolism in Parkinson's disease without dementia. Neurobiol Aging. 1994;15:329-35.

41. Mejias R, Villadiego J, Pintado CO, Vime PJ, Gao L, Toledo-Aral JJ, Echevarria M, Lopez-Barneo J. Neuroprotection by transgenic expression of glucose-6phosphate dehydrogenase in dopaminergic nigrostriatal neurons of mice. $J$ Neurosci. 2006:26:4500-8.

42. Anandhan A, Lei S, Levytskyy R, Pappa A, Panayiotidis Ml, Cerny RL, Khalimonchuk O, Powers R, Franco R. Glucose metabolism and AMPK signaling regulate dopaminergic cell death induced by gene (alphaSynuclein)-environment (Paraquat) interactions. Mol Neurobiol. 2017:54: 3825-42.

43. Lei S, Zavala-Flores L, Garcia-Garcia A, Nandakumar R, Huang Y, Madayiputhiya N, Stanton RC, Dodds ED, Powers R, Franco R. Alterations in energy/redox metabolism induced by mitochondrial and environmental toxins: a specific role for glucose-6-phosphate-dehydrogenase and the 
pentose phosphate pathway in paraquat toxicity. ACS Chem Biol. 2014;9:2032-48.

44. Spaans SK, Weusthuis RA, van der Oost J, Kengen SW. NADPH-generating systems in bacteria and archaea. Front Microbiol. 2015;6:742.

45. Jain M, Brenner DA, Cui L, Lim CC, Wang B, Pimentel DR, Koh S, Sawyer DB, Leopold JA, Handy DE, et al. Glucose-6-phosphate dehydrogenase modulates cytosolic redox status and contractile phenotype in adult cardiomyocytes. Circ Res. 2003;93:e9-16.

46. Leopold JA, Zhang YY, Scribner AW, Stanton RC, Loscalzo J. Glucose-6phosphate dehydrogenase overexpression decreases endothelial cell oxidant stress and increases bioavailable nitric oxide. Arterioscler Thromb Vasc Biol. 2003;23:411-7.

47. Nicol CJ, Zielenski J, Tsui LC, Wells PG. An embryoprotective role for glucose-6-phosphate dehydrogenase in developmental oxidative stress and chemical teratogenesis. Faseb j. 2000;14:111-27.

48. Park J, Chung JJ, Kim JB. New evaluations of redox regulating system in adipose tissue of obesity. Diabetes Res Clin Pract. 2007;77(Suppl 1):S11-6.

49. Gupte SA, Levine RJ, Gupte RS, Young ME, Lionetti V, Labinskyy V, Floyd BC, Ojaimi C, Bellomo M, Wolin MS, Recchia FA. Glucose-6-phosphate dehydrogenase-derived NADPH fuels superoxide production in the failing heart. J Mol Cell Cardiol. 2006:41:340-9.

50. Matsui R, Xu S, Maitland KA, Hayes A, Leopold JA, Handy DE, Loscalzo J, Cohen RA. Glucose-6 phosphate dehydrogenase deficiency decreases the vascular response to angiotensin II. Circulation. 2005;112:257-63.

51. Matsui R, Xu S, Maitland KA, Mastroianni R, Leopold JA, Handy DE, Loscalzo J, Cohen RA. Glucose-6-phosphate dehydrogenase deficiency decreases vascular superoxide and atherosclerotic lesions in apolipoprotein $\mathrm{E}(-/-)$ mice Arterioscler Thromb Vasc Biol. 2006;26:910-6.

52. Park J, Rho HK, Kim KH, Choe SS, Lee YS, Kim JB. Overexpression of glucose6-phosphate dehydrogenase is associated with lipid dysregulation and insulin resistance in obesity. Mol Cell Biol. 2005;25:5146-57.

53. Serpillon S, Floyd BC, Gupte RS, George S, Kozicky M, Neito V, Recchia F, Stanley W, Wolin MS, Gupte SA. Superoxide production by NAD(P)H oxidase and mitochondria is increased in genetically obese and hyperglycemic rat heart and aorta before the development of cardiac dysfunction. The role of glucose-6-phosphate dehydrogenase-derived NADPH. Am J Physiol Heart Circ Physiol. 2009;297:H153-62.

54. Gupte RS, Floyd BC, Kozicky M, George S, Ungvari ZI, Neito V, Wolin MS, Gupte SA. Synergistic activation of glucose-6-phosphate dehydrogenase and $\mathrm{NAD}(\mathrm{P}) \mathrm{H}$ oxidase by Src kinase elevates superoxide in type 2 diabetic, Zucker fa/fa, rat liver. Free Radic Biol Med. 2009;47:219-28.

55. Lee JW, Choi AH, Ham M, Kim JW, Choe SS, Park J, Lee GY, Yoon KH, Kim JB. G6PD up-regulation promotes pancreatic beta-cell dysfunction. Endocrinology. 2011;152:793-803.

56. Ham M, Lee JW, Choi AH, Jang H, Choi G, Park J, Kozuka C, Sears DD, Masuzaki H, Kim JB. Macrophage glucose-6-phosphate dehydrogenase stimulates proinflammatory responses with oxidative stress. Mol Cell Biol. 2013;33:2425-35.

57. Ham M, Choe SS, Shin KC, Choi G, Kim JW, Noh JR, Kim YH, Ryu JW, Yoon KH, Lee CH, Kim JB. Glucose-6-phosphate dehydrogenase deficiency improves insulin resistance with reduced adipose tissue inflammation in obesity. Diabetes. 2016;65:2624-38.

58. Voloboueva LA, Emery JF, Sun X, Giffard RG. Inflammatory response of microglial BV-2 cells includes a glycolytic shift and is modulated by mitochondrial glucose-regulated protein $75 /$ mortalin. FEBS Lett. 2013;587:756-62

59. Gimeno-Bayon J, Lopez-Lopez A, Rodriguez MJ, Mahy N. Glucose pathways adaptation supports acquisition of activated microglia phenotype. J Neurosci Res. 2014:92:723-31.

60. Orihuela R, McPherson CA, Harry GJ. Microglial M1/M2 polarization and metabolic states. Br J Pharmacol. 2016;173:649-65.

61. Requejo-Aguilar R, Bolanos JP. Mitochondrial control of cell bioenergetics in Parkinson's disease. Free Radic Biol Med. 2016;100:123-37.

62. Falkowska A, Gutowska I, Goschorska M, Nowacki P, Chlubek D, BaranowskaBosiacka I. Energy metabolism of the brain, including the cooperation between astrocytes and neurons, especially in the context of glycogen metabolism. Int J Mol Sci. 2015;16:25959-81.

63. Bolanos JP. Bioenergetics and redox adaptations of astrocytes to neuronal activity. J Neurochem. 2016;139(Suppl 2):115-25.
64. Volkenhoff A, Weiler A, Letzel M, Stehling M, Klambt C, Schirmeier S. Glial glycolysis is essential for neuronal survival in drosophila. Cell Metab. 2015 22:437-47.

65. Lee Y, Morrison BM, Li Y, Lengacher S, Farah MH, Hoffman PN, Liu Y, Tsingalia A, Jin L, Zhang PW, et al. Oligodendroglia metabolically support axons and contribute to neurodegeneration. Nature. 2012;487:443-8.

66. Bubp J, Jen M, Matuszewski K. Caring for glucose-6-phosphate dehydrogenase (G6PD)-deficient patients: implications for pharmacy. P t. 2015:40:572-4.

\section{Publisher's Note}

Springer Nature remains neutral with regard to jurisdictional claims in published maps and institutional affiliations.

\section{Ready to submit your research? Choose BMC and benefit from:}

- fast, convenient online submission

- thorough peer review by experienced researchers in your field

- rapid publication on acceptance

- support for research data, including large and complex data types

- gold Open Access which fosters wider collaboration and increased citations

- maximum visibility for your research: over $100 \mathrm{M}$ website views per year

At BMC, research is always in progress.

Learn more biomedcentral.com/submissions 